\title{
Análise da superfície de implantes odontológicos submetidos a ensaio de torção
}

\author{
Surface analysis of dental implants tested in torsion
}

Gabriela Benedini Strini Portinari Beja* Mariana Lima da Costa Valente*

Ana Beatriz Vilela Teixeira* Jonas Guilherme Gomes Wizel*** Andréa Candido dos Reis ${ }^{* * * *}$

\section{Resumo}

Objetivo: Avaliar o comportamento mecânico de implantes odontológicos submetidos à torção manual, a fim de analisar o ponto de ruptura e determinar a região mais frágil e susceptível à deformação, por meio de microscopia eletrônica de varredura, antes e após a realização do ensaio de torção. Materiais e método: Foram selecionados quatro modelos de implantes da marca Conexão ${ }^{\circledast}(n=5)$ : Master Screw, Porous, Conect AR e Conect Cônico. Foi utilizado um torquímetro digital para a aplicação de torque e com um microscópio eletrônico de varredura avaliou-se a superfície dos implantes antes e após o ensaio de torção. Resultados: Os resultados foram obtidos pela análise estatística ANOVA $(P<0,05)$, seguido do teste de Tukey $(P<0,05)$. Após a torção verificou-se que as quatro amostras de implantes apresentaram diferentes médias para a variável força máxima, porém, sem diferença estatística significante para os implantes Master Screw, Porous e Conect AR ( $p>0,05)$, sendo apenas o implante Conect Cônico estatisticamente diferente $(p<0,05)$. Quanto às imagens obtidas a partir da análise ao MEV observou-se fratura do montador dos implantes Master Screw e Porous, ruptura dos implantes Conect $A R$, e espanamento das roscas internas dos Cônicos. Conclusão: Os modelos de implantes testados apresentaram diferentes comportamentos mecânicos quando submetidos à aplicação de forças, com os Cônicos apresentando menor resistência ao ensaio de torção.

Palavras-chave: Torção mecânica. Microscopia eletrônica de varredura. Implantes dentários.

\section{Introdução}

O uso de implantes é reconhecido como um tratamento previsível e bem sucedido para restaurar a função total ou parcial de um paciente desdentado ${ }^{1}$ e o seu sucesso a curto ou longo prazo está relacionado a diversos fatores como geometria, composição, tamanho e morfologia superficial, os quais associados a uma técnica cirúrgica adequada interferem na estabilidade primária e, consequentemente, na osseointegração, um dos pré-requisitos para o sucesso clínico das reabilitações orais com implantes ${ }^{2,3}$.

A morfologia superficial dos implantes modula sua ancoragem e resposta óssea ${ }^{4}$, por isso, alterações, como a criação de micro rugosidades na superfície dos implantes têm sido realizadas, a fim de acelerar o processo de cicatrização do osso e aumentar a aposição óssea ao redor dos parafusos de implantes ${ }^{5,6}$.

Para avaliar os diferentes tipos de implantes disponíveis no mercado, os ensaios mecânicos são fundamentais, pois, por meio deles, é possível analisar o desempenho do material testado, quando submetido a diferentes tensões e esforços, em diferentes tipos de substratos ${ }^{7}$. Esses ensaios permitem predizer a durabilidade do parafuso, quantificar o torque de inserção e o nível de adaptação prótese/ implante, através da análise da plataforma protéti-

Graduados em Odontologia pela Faculdade de Odontologia de Ribeirão Preto/USP, São Paulo, SP, Brasil.

Mestranda na Área de Reabilitação Oral da Faculdade de Odontologia de Ribeirão Preto/USP, São Paulo, SP, Brasil.

Graduado pela Universidade Federal de São Carlos, Departamento de Engenharia de Produção, São Carlos, SP, Brasil.

Professora Doutora do Departamento de Materiais Dentários e Prótese da Faculdade de Odontologia de Ribeirão Preto FORP-USP, São Paulo, SP, Brasil. 
ca desses ${ }^{8}$, já que, segundo a literatura, os maiores problemas no sucesso de uma reabilitação oral com próteses implantossuportadas estão relacionados aos diferentes formatos, às plataformas protéticas e aos tratamentos de superfície ${ }^{9}$.

Um resultado clínico satisfatório também depende da capacidade do implante de suportar as cargas oriundas da cavidade bucal, importante para a manutenção do parafuso no leito ósseo a longo prazo. Para uma distribuição correta de forças no tecido ósseo é importante que haja estabilidade entre a prótese e o implante, por meio da plataforma protética, para que o excesso de tensões não provoque a falha do implante e consequentemente o insucesso da reabilitação oral ${ }^{10,12}$.

Assim, a proposta do estudo foi avaliar a superfície de implantes de diferentes formatos e tratamentos de superfícies antes e após realização de ensaio de torção, por meio de microscopia eletrônica de varredura (MEV), de modo a verificar alterações na morfologia dos implantes que possam levar ao prejuízo da osseointegração e ao insucesso do tratamento reabilitador.

\section{Materiais e método}

Foram utilizados vinte implantes da marca Conexão ${ }^{\circledR}$ (Arujá, São Paulo, Brasil) $(\mathrm{n}=5)$ : Master Screw, cilíndrico de superfície usinada $(\varnothing 3,75$ mm x $11,5 \mathrm{~mm}$ ), Porous, cilíndrico com duplo tratamento de superfície Porous ( $\varnothing$ 3,75 mm x 11,5 mm), Conect AR, cilíndrico com tratamento de superfície Porous (Ø 3,75 mm x 11,5 mm), Conect Cônico, cônico com tratamento de superfície Porous (Ø 3,5 mm x 11,5 $\mathrm{mm}$ ). Todos os implantes foram submetidos à análise microscópica, por meio de microscopia eletrônica de varredura e ao ensaio de torção manual.

\section{Ensaio de torção manual:}

O ensaio de torção manual foi realizado com um torquímetro digital Mackena ${ }^{\circledR}$ (São Paulo - SP, Brasil), modelo MK-20XX, com capacidade de 10 N.m e precisão de $0,01 \mathrm{~N} . \mathrm{m}$, para a quantificação do torque imposto ao parafuso a cada angulação. Os parafusos foram posicionados em uma morsa, mantendo seu recesso hexagonal voltado para cima, também foram deixados cinco fios de rosca livres, como exige a NBR ISO 6475:199713.

Com a peça de encaixe hexagonal do torquímetro acoplada ao sextavado da cabeça do parafuso, seguindo os padrões especificados da NBR ISO 8319-1:1998 ${ }^{14}$, mantendo um ângulo de $90^{\circ}$ entre o braço do torquímetro e o eixo longitudinal do parafuso, iniciou-se movimento circular, em sentindo horário, com velocidade dentro dos valores exigidos (1 a 5 rotações/minutos), até o momento de torção do implante, com ou sem ruptura.

Os quatro grupos de implantes foram submetidos ao ensaio de torção, com a finalidade de analisar a força máxima suportada pelo implante.

\section{Microscopia Eletrônica de Varredura:}

Para uma análise detalhada da superfície dos implantes, foi usado o equipamento microscópio eletrônico de varredura JSM- 5200 (JEOL Ltd, Tóquio, Japão), com aumento de 35 vezes. As fotomicrografias foram feitas com um filme fotográfico preto e branco, 120 mm (Kodak ${ }^{\circledR}$, Tmáx 100, Rochester, New York, Estados Unidos). Os implantes foram posicionados no tubo de incisão de feixes de elétrons do microscópio na posição horizontal, mantendo exposta a sua superfície superior. A varredura da superfície foi projetada em um monitor com a imagem ampliada da superfície do implante, permitindo analisar a rosca do implante e suas demais estruturas ${ }^{15}$.

\section{Resultados}

Para a análise estatística foi utilizado o software Minitab versão 15.1.1.0. Verificada a normalidade do estudo utilizou-se ANOVA, para análise de variância, seguido do teste de Tukey, com $5 \%$ de nível de significância, para comparação das médias.

De acordo com o teste ANOVA, os quatro implantes apresentaram diferentes médias de força máxima de torção $p=0,00$. Quando comparados entre si verificou-se semelhança estatística entre os implantes Master Screw, Master Porous e Conect AR $(p>0,05)$, e diferença estatística do implante Conect Cônico com os demais implantes testados ( $p$ $<0,05$ ) (Tab. 1).

Tabela 1 - Média e desvio padrão dos implantes (n=5) para a força máxima de torção (N.cm)

\begin{tabular}{l|c|c}
\hline \multicolumn{1}{c|}{ Implante } & Força Máxima de Torção (N.cm) & Valor de $p$ \\
\hline Master Screw & $125,4 \pm 0,217^{\mathrm{A}}$ & $p>0.05$ \\
Master Porous & $129,8 \pm 0,294^{\mathrm{A}}$ & $p>0,05$ \\
Conect AR & $149,8 \pm 0,327^{\mathrm{A}}$ & $p>0,05$ \\
Conect Cônico & $54,4 \pm 0,089^{\mathrm{B}}$ & $p>0,05$
\end{tabular}

*Letras iguais significam semelhança estatística e letras diferentes, diferença estatística. 
Quando analisados no MEV, os implantes de um mesmo grupo apresentaram superfície nítida e sem modificações prévias ao ensaio de torção. Após a aplicação da força máxima, observaram-se alterações morfológicas nos parafusos ensaiados, que va- riaram de acordo com o modelo de implante testado. Os implantes Master Screw e Porous apresentaram fratura do montador (Fig. 1 e 2), os Conect AR fratura do implante (Fig. 3) e os Conect Cônico deformação das roscas internas (Fig. 4).
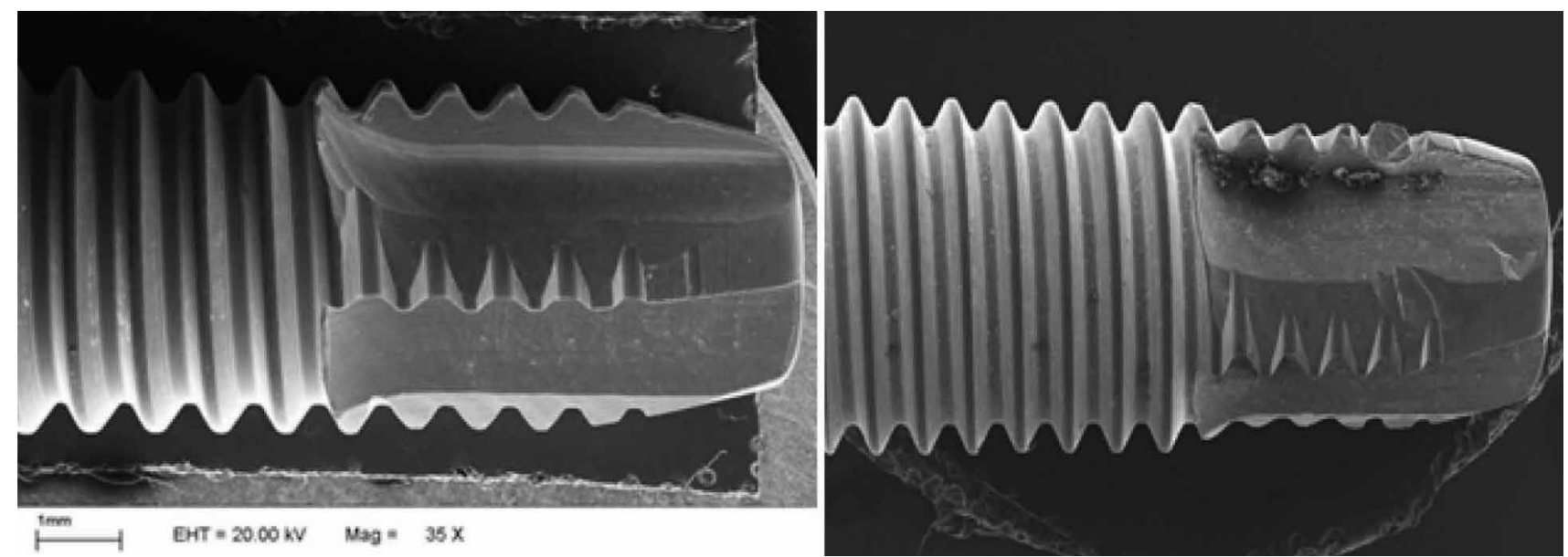

Figura 1 - Implante Master Screw antes e após ensaio de torção

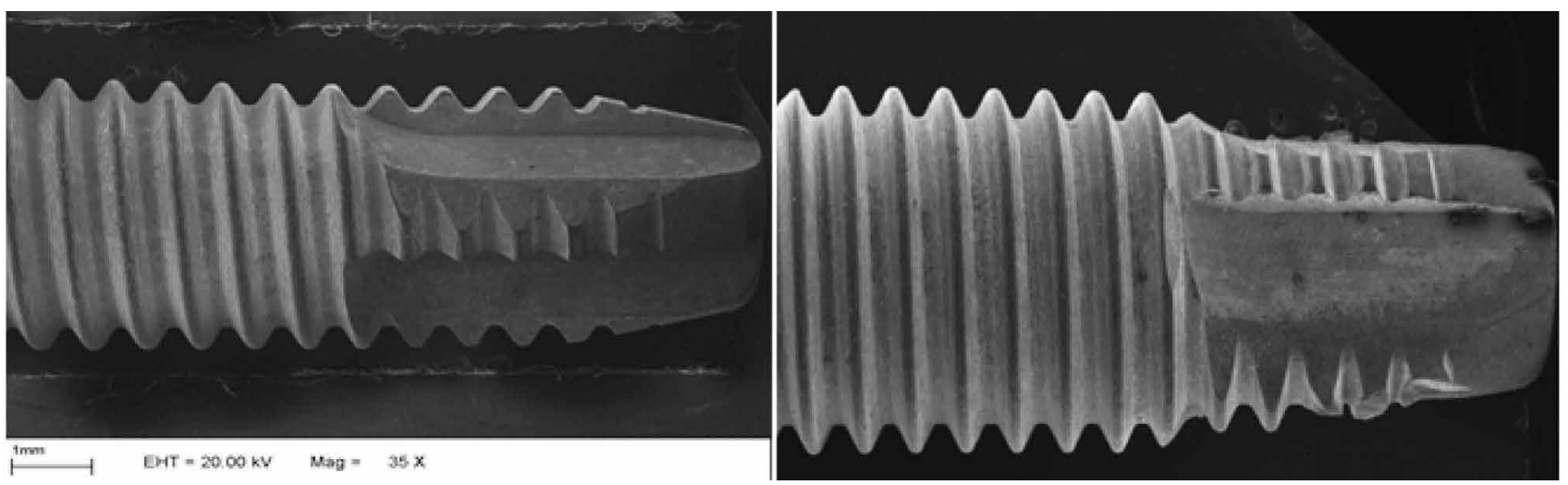

Figura 2 - Implante Porous antes e após ensaio de torção
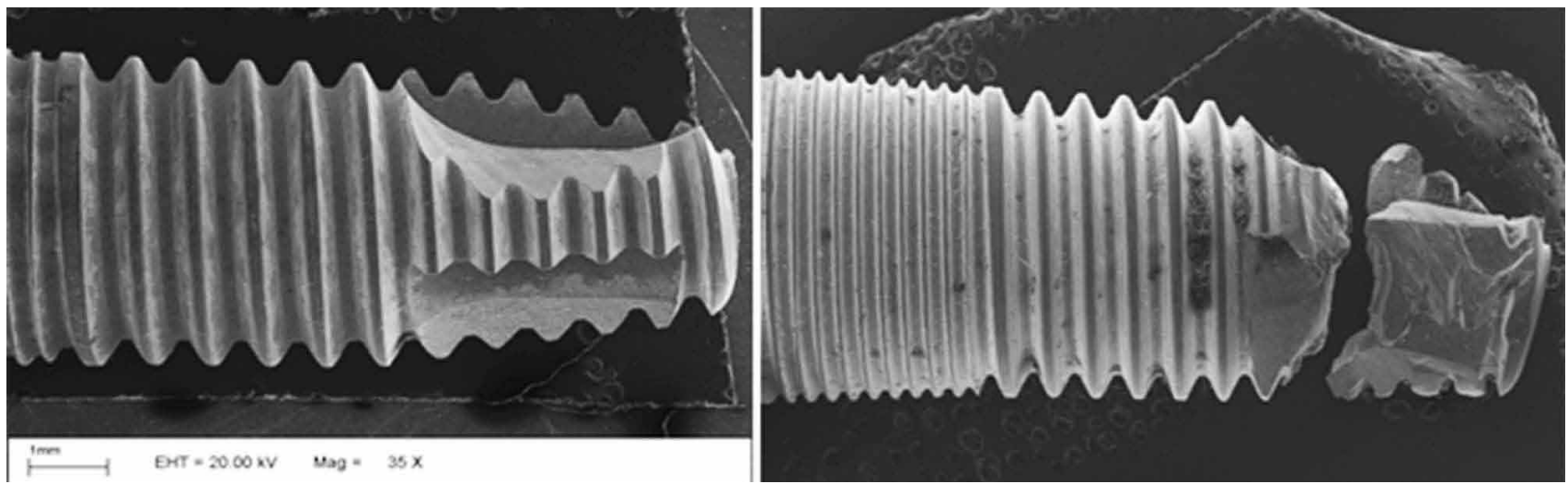

Figura 3 - Implante Conect AR antes e após ensaio de torção 


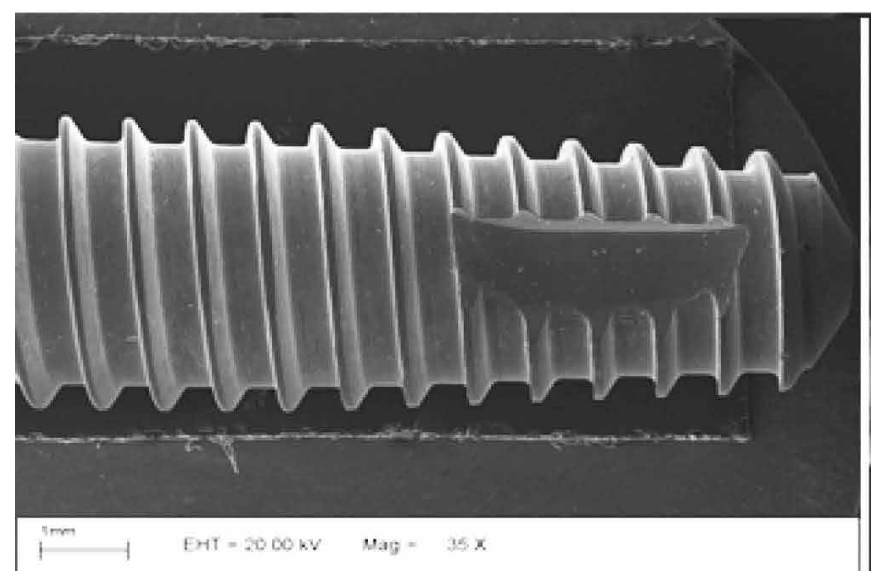

Figura 4 - Implante Conect Cônico antes e após ensaio de torção

\section{Discussão}

Devido a grande taxa de sucesso das reabilitações orais com próteses implantossuportadas, cada vez mais os implantes vem sendo utilizados como alternativa de tratamento para pacientes desdentados totais e parciais. Entretanto, inadequações mecânicas podem ocorrer, como desadaptações protéticas na interface osso/implante, deformações da plataforma protética, excesso de tensões transmitidas ao tecido ósseo e a fratura do próprio implante ${ }^{16-17}$.

Os diferentes formatos de implantes e tipos de plataformas protéticas reagem de maneira diferente à aplicação de tensões. Segundo a literatura ${ }^{18}$, implantes de hexágono externo instalados com a utilização de montadores, que aplicam carga diretamente na conexão, estão sujeitos a deformações na região do hexágono quando o torque for superior a 55 N.cm e sujeitos ao completo espanamento desse, com torques próximos a 70 N.cm. Por outro lado, implantes com dispositivos de instalação interna, viabilizam a aplicação de torques próximos a 80 N.cm, sem provocar deformações na plataforma protética e sem demonstrar indício clínico ou radiográfico de necrose óssea decorrente da aplicação do torque ${ }^{19}$.

No estudo, a aplicação de forças durante o ensaio de torção provocou diferentes alterações na estrutura dos implantes. No modelo Master Screw ocorreu a ruptura do montador com aplicação de força de 125,4 N.cm, já no implante Porous, a ruptura se deu com 129,8 N.cm. O implante Conect AR fraturou na região de corte com aplicação de torque de 149,8 N.cm e o Cônico teve suas roscas internas espanadas com um torque de 54,4 N.cm. Os implantes Conect AR apresentaram os maiores valores de força máxima de torção, possivelmente devido à plataforma protética do tipo hexágono interno longo que direciona a transferência da força de tração para o longo eixo do parafuso.

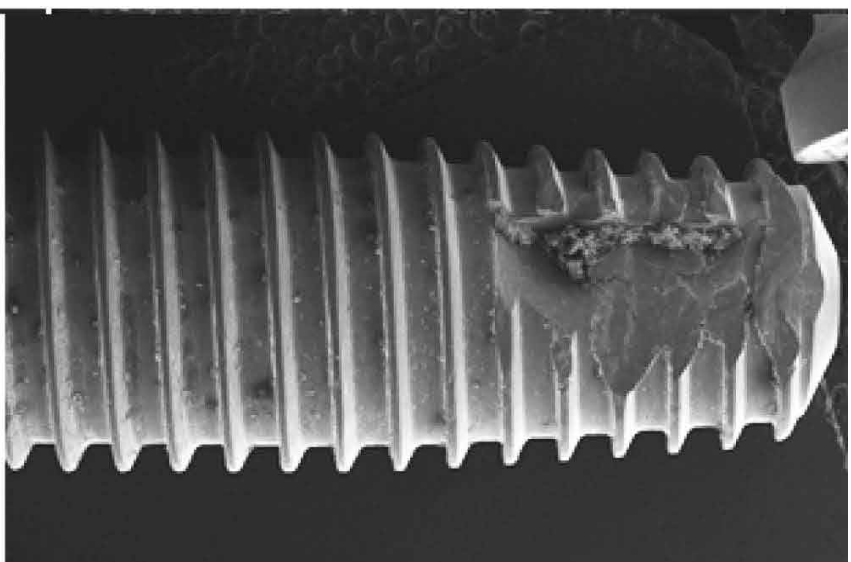

Por outro lado, os implantes Conect Cônico apresentaram os menores valores de torção, 54 N.cm, com o espaçamento das roscas, provavelmente devido a sua configuração interna, com uma rosca muito estreita, o que facilita a deformação dessas e impede a adequada dissipação de tensões ao longo eixo do parafuso.

A fratura do componente protético, tão comum nos casos de insucesso de reabilitações orais com implantes ${ }^{20-21}$, ocorre principalmente devido ao afrouxamento não detectado do componente, somado aos movimentos não axiais durante o carregamento protético, podendo ocorrer ao nível da porção superior hexagonal, como observado nos implantes Porous e Master Screw, que apresentaram a ruptura do componente (montador), na porção interna do implante, como observado nos implantes Conect Cônico e Conect AR.

O elevado estresse mecânico gerado nos componentes pode provocar a desadaptação da prótese e até a fratura do implante ${ }^{22}$, como no caso do Conect AR, que não suportou a carga aplicada sobre o montador, o que levou a ruptura do parafuso. Já o implante Conect Cônico teve suas roscas internas espanadas, ou seja, em uma situação clínica isso iria gerar uma folga e consequente desadaptação na interface protética ${ }^{23}$.

$\mathrm{O}$ presente estudo mostrou que diferentes tipos de implantes reagem distintamente à aplicação de forças e, também, que as características morfológicas como geometria, tamanho plataforma protética influenciam diretamente na distribuição de forças ao longo do parafuso. Por isso, diante da diversidade de implantes disponíveis no mercado odontológico, é fundamental que o cirurgião dentista tome conhecimento prévio do material que irá utilizar em uma reabilitação oral com próteses implantossuportadas. 


\section{Conclusão}

Os implantes testados apresentaram diferentes comportamentos mecânicos após aplicação da força máxima de torção. No estudo, os implantes cônicos, quando comparados aos cilíndricos apresentaram menor resistência ao ensaio de torção.

\section{Abstract}

Objective: To evaluate the mechanical behavior of dental implants subjected to manual torsion in order to analyze the breaking point and determine the region more fragile and susceptible to deformation by means of scanning electron microscopy before and after completion of the torsion test. Materiais and method: It was selected four models of the brand Connection ${ }^{\circledR}$ implants $(n=5)$ : Master Screw, Porous, Conect AR and Conect Conical. We used a digital torque wrench for applying torque and with a scanning electron microscope evaluated the implant surface before and after the torsion test. Results: The results were obtained by statistical analysis ANOVA $(P<0.05)$, followed by Tukey test $(P<0.05)$. After the twist was found that the four samples showed different implants averages for maximum strength, however, no statistically significant difference for the Master Screw, Porous and Conect AR implants $(P>0,05)$, only the implant Conect Conical statistically different $(P<0,05)$. As for the images obtained from the SEM analysis it was observed assembler fracture implants Master Screw e Porous, rupture of implants Conect AR and dusted the internal threads of the Connect Conical. Conclusion: The models of implants tested showed different behaviors when subjected to mechanical force application, with tapered presenting less resistance to the torsion test.

Keywords: Torsion mechanical. Scanning electron microscopy. Dental implants.

\section{Referências}

1. Albrektsson T, Branemark PI, Hansson HA, Lidstrom J. Osseointegrated Titanium Implants: Requirements for Ensuring a Long-Lasting, Direct Bone-to-Implant Anchorage in Man. Acta Orthop Scand 1981; 52(2):155-70.

2. Le. Guéhennec L, Soueidan A, Layrolle P, Amouriq Y. Surface treatments of titanium dental implants for rapid osseointegration. Dent Mater 2007; 23(7):844-54.

3. Ratner BD. Surface characterization of biomaterials by electron spectroscopy for chemical analysis. Ann Biomed Eng $1983 ; 11(3-4): 313-36$.

4. Beck U, Lange R, Neumann HG. Micro-plasma textured Ti-implant surfaces. Biomol Eng 2007; 24(1):47-51.

5. Kim K, Lee BA, Piao XH, Chung HJ, Kim YJ. Surface characteristics and bioactivity of an anodized titanium surface. J Periodontal Implant Sci 2013; 43(4)198-205.

6. Mazzo CR, Reis AC, Shimano AC, Valente ML. In vitro analysis of the influence of surface treatment of dental implants on primary stability. Braz Oral Res 2012; 26(4):313-7.
7. Oliscovicz NF, Valente MLC, Marcantonio Jr E, Shimano $\mathrm{AC}$, Reis AC. Estudo in vitro da influência do formato e do tratamento de superfície de implantes odontológicos no torque de inserção, resistência ao arrancamento e frequência de ressonância. Rev Odontol UNESP 2013; 42(4):283-90.

8. Tjellstrom A, Lindstrom J, Hallén O, Albrektsson T, Branemark PI. Osseointegrated titanium implants in the temporal bone. A clinical study on bon-anchored hearing aids. Am J Otol 1981; 2(4):304-10.

9. Binon PP. Implants and components: entering the new millennium. Int J Oral Maxillofac Implants 2000; 15(1):76-94.

10. Huang YM, Chou IC, Jiang CP, Wu YS, Lee SY. Finite element analysis of dental implant neck effects on primary stability and osseointegration in a type IV bone mandible. Biomed Mater Eng 2014; 24(1):1407-15.

11. Jesus Tavarez RR, Bonachela WC, Xible AA. Effect of cyclic load on vertical misfit of prefabricated and cast implant single abutment. J Appl Oral Sci 2011; 19(1):16-21.

12. Pessoa RS, Muraru L, Júnior EM, Vaz LG, Sloten JV, Duyck $\mathrm{J}$, Jaecques SV. Influence of implant connection type on the biomechanical environment of immediately placed implants - CT - based nonlinear, three-dimensional finite element analysis. Clin Implant Dent Relat Res 2010; 12(3):219-34.

13. Associação Brasileira de Normas Técnicas - NBR ISO 6475. Implantes para cirurgia - parafusos ósseos metálicos com rosca assimétrica e superfície inferior da cabeça de forma esférica - requisitos mecânicos e métodos de ensaio, 1987.

14. Associação Brasileira de Normas Técnicas - NBR ISO 83191. Instrumentos Ortopédicos - conexões de ferramenta. Parte 1: chaves para uso com parafusos com encaixe hexagonal na cabeça; 1998 .

15. Shimano SGN, Shimano AC. Comportamento de parafusos corticais submetidos a ensaio de torção manual e de torção em máquina. Acta ortop bras 2008; 16(2):81-4.

16. Aguiar RC, Scherer D, Battisti TC, Gassen HT, Silva AN. Fratura de implante dentário: relato de caso clínico. Stomatos $2007 ; 13(24): 37-44$.

17. Hecker D, Eckert SE, Choi YG. Cyclic loading of implantsupported prostheses: comparison of gaps at the prosthetic-abutment interface when cycled abutments are replaced with as- manufactured abutments. J Prosthet Dent 2006; 95(1):26-32.

18. Constantino A. Osseocompressão: otimizando a estabilidade primária para a ativação imediata de implantes. ImplantNews 2004;1(3):219-226, 2004

19. Rocha FA, Elias CN. Influência da técnica cirúrgica e da formado implante na estabilidade primária. Rev Odontol Bras Central 2010; 18(48):26-9.

20. Branemark PI, Adell R, Breine U, Hansson BO, Lindstrom $\mathrm{J}$, Ohlsson A. Intra-osseous anchorage of dental prostheses. I. Experimental studies. Scand J Plast Reconstr Surg. 1969; 3(2):81-100.

21. Rivaldo EG, Wutke C, Silveira M, Frasca LCF, Ferandes EL, Pocztaruk RL. Falhas estruturais em prótese total fixa sobre implantes: relato de caso clínico. Stomatos. 2007; 13(25):131-8.

22. Vasconcellos DK, Bottino MA, Nishioka RS, Valandro LF, Costa EM. The influence of different Screw tightening forces on the vertical misfit of implant-supported frameworks. J Appl Oral Sci 2005; 13(2):120-5. 
23. Cibirka RM, Nelson SK, Lang BR, Rueggeberg FA. Examination of the implant-abutment interface after fatigue testing. J Prosthet Dent 2001; 85(3):268-75.

\section{Endereço para correspondência:}

Andréa Candido dos Reis

Av. do Café, $\mathrm{s} / \mathrm{n}^{\circ}$

14040-904 Ribeirão Preto/SP

Fone: 551636024790

E-mail: andreare@forp.usp.br e andreare73@yahoo.com

Recebido: 10/11/2013. Aceito: 27/03/2014. 